\title{
Tailoring the Performance of Organic Solvent Nanofiltration Membranes with Biophenol Coatings
}

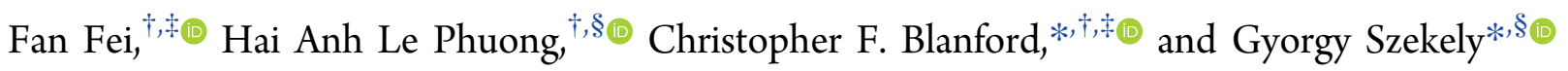 \\ ${ }^{\dagger}$ School of Materials, University of Manchester, Oxford Road, Manchester, M13 9PL, United Kingdom \\ ${ }^{\ddagger}$ Manchester Institute of Biotechnology, University of Manchester, 131 Princess Street, Manchester M1 7DN, United Kingdom \\ ${ }^{\S}$ School of Chemical Engineering and Analytical Science, University of Manchester, The Mill, Sackville Street, Manchester M1 3BB, \\ United Kingdom
}

\section{Supporting Information}

ABSTRACT: This study reports a systematic investigation of fine-tuning the filtration performance of nanofiltration membranes with biophenol coatings to produce solvent-resistant membranes with $390-1550 \mathrm{~g} \mathrm{~mol}^{-1}$ molecular weight cutoff (MWCO) and $0.5-40 \mathrm{~L} \mathrm{~m}^{-2} \mathrm{~h}^{-1} \mathrm{bar}^{-1}$ permeance. Six kinds of inexpensive, commercial biophenols (dopamine, tannic acid, vanillyl alcohol, eugenol, morin, and quercetin) were subjected to identical oxidant-promoted polymerization to coat six kinds of loose asymmetric membrane supports: polyimide (PI), polyacrylonitrile (PAN), polysulfone (PSf), polyvinylidene difluoride

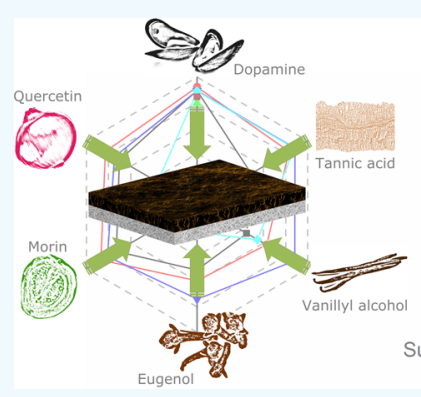
Accessible materials (PVDF), polybenzimidazole (PBI), and polydimethylsiloxane (PDMS). The coatings were characterized by Fourier-transform infrared spectroscopy (FTIR), and the morphologies were characterized by scanning electron microscopy (SEM) and atomic force microscopy (AFM). The long-term stability of 42 membranes were tested in 12 organic solvents, including emerging green solvents MeTHF and Cyrene. The biophenol coatings led to tighter membranes with a decrease in $\mathrm{MWCO}$ of $12-79 \%$ at a penalty of a $22-92 \%$ permeance decrease in acetone.

KEYWORDS: surface modification, oxidant-promoted polymerization, phase inversion, membrane separation, asymmetric membranes

\section{INTRODUCTION}

Organic solvent nanofiltration (OSN) is a pressure-driven separation process with a great potential in petrochemical and pharmaceutical purifications. It allows separations at the molecular level between 50 and $2000 \mathrm{~g} \mathrm{~mol}^{-1}$ in organic media. ${ }^{1}$ OSN is an energy-efficient technology compared to other separation techniques, such as distillation, recrystallization, chromatography, and extraction. ${ }^{2,3}$

Compared with ceramics, glasses, and metals, polymers have several advantages in fabricating OSN membranes, namely, higher pliability, lower cost, facile processability, and scalability. Among various membrane fabrication techniques, phase inversion by immersion precipitation, i.e., nonsolventinduced phase inversion, has been widely used for preparing nanofiltration membranes. ${ }^{4,5}$ Phase inversion leads to an integrally skinned asymmetric membrane with a dense top layer providing separation properties and a porous sublayer providing mechanical support. Various polymers, such as polyimide (PI), ${ }^{6}$ polyacrylonitrile (PAN), ${ }^{7}$ polysulfone (PSf), ${ }^{8}$ polyvinylidene difluoride (PVDF), ${ }^{9}$ polybenzimidazole (PBI), ${ }^{10}$ poly(ether-ether-ketone) (PEEK), ${ }^{11}$ polyoxindolebiphenylene (POX), ${ }^{12}$ polyarylene sulfide sulfone (PASS), ${ }^{13}$ and sulfonated polyarylene ether sulfone (SPAES), ${ }^{14}$ have been used to make OSN membranes by phase inversion.
The overall separation performance of OSN membranes is mainly determined by the membrane's dense top layer, which can be customized by various surface modification techniques, such as dip coating, spin coating, polymer grafting, plasma treatment, polyelectrolyte deposition, and interfacial polymerization. ${ }^{15}$ Mussel-inspired surface modification chemistry has been intensively studied by the membrane community ${ }^{16-23}$ since the Messersmith's group seminal work on the use of the self-polymerization of dopamine as a bioinspired, versatile, and material-independent coating technique. ${ }^{24}$ Dopamine, a catecholamine (Figure S2), has been the most common monomer used in membrane coatings. ${ }^{18}$ Dopamine is identical to the hydroxylated tyrosine residues found in mussel foot proteins that produce the strong adhesion of mussels onto wet surfaces. ${ }^{18}$ Under oxidative, weakly alkaline conditions, dopamine can self-polymerize to polydopamine and can deposit onto a variety of surfaces at ambient temperature. ${ }^{24}$

Owing to their structural resemblance to dopamine, plantbased biophenols are low-cost alternatives for bioinspired membrane coatings (Figure 1). ${ }^{19-21}$ Members of these

Received: November 23, 2018

Accepted: January 29, 2019

Published: January 29, 2019 


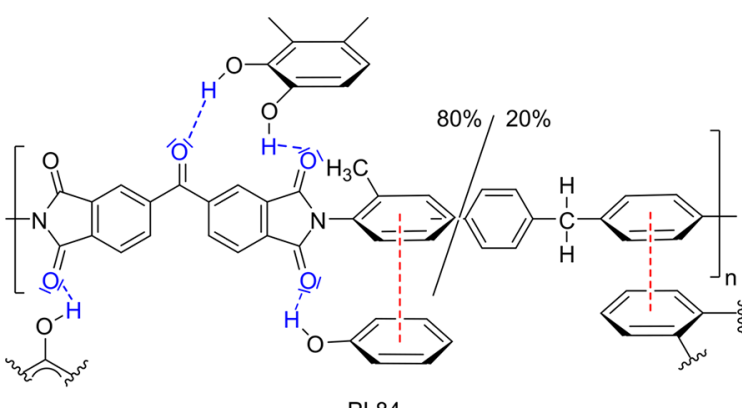

$\mathrm{PI} 84$

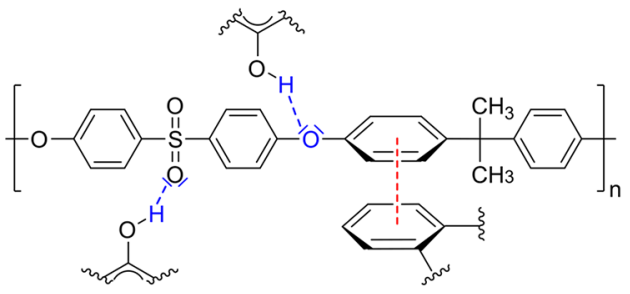

PSf

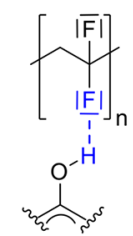

PVDF

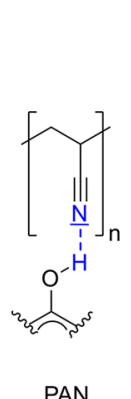

PAN

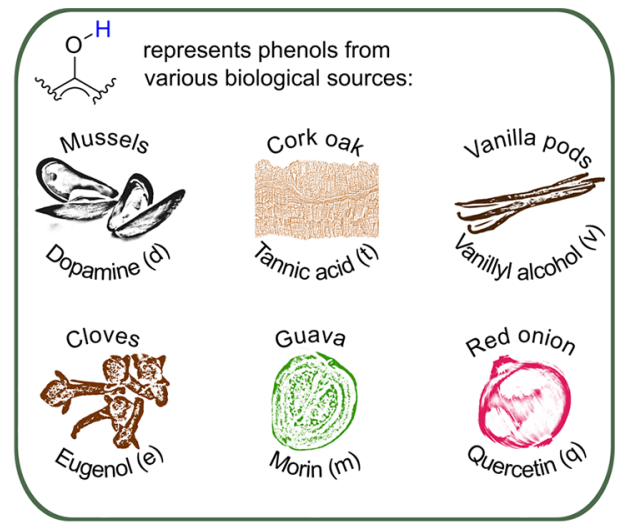

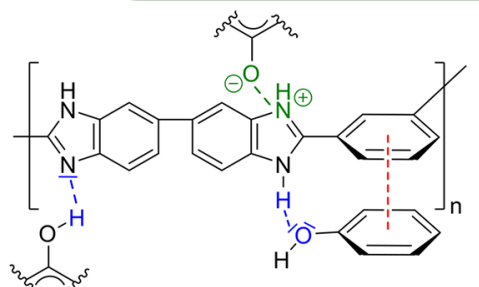

PBI

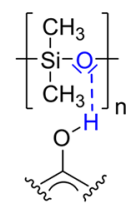

PDMS

------ H-bond

$\mathrm{pH}$ dependent electrostatic interaction

-.-- p-p stacking

Figure 1. Possible chemical interactions between the applied polymers and catechol moiety of polyphenols. PI, polyimide; PAN, polyacrylonitrile; PSf, polysulfone; PVDF, polyvinylidene difluoride; PBI, polybenzimidazole; and PDMS, polydimethylsiloxane.

compounds involve tannic acid (typically sourced from seeds and galls), vanillyl alcohol (commonly produced from lignin waste), eugenol (extracted from cloves), morin (from guava leaves), and quercetin (a common plant flavanol) (Figure S2). Apart from morin, the biophenol coating precursors are 4095\% less expensive compared to dopamine (Table S1).

Polyphenols and their monomers have abundant catechol and pyrogallol functionalities that allow for binding to the membrane surface through the formation of hydrogen bonds or through $\pi-\pi$ stacking of aromatic moieties (Figure 1). Polyphenols' acidic - $\mathrm{OH}$ groups can also bind to charged materials through electrostatic interactions and can also chelate metal ions depending on the $\mathrm{pH}$ of the binding environment. In addition, the catechol moiety of polyphenols can undergo $\mathrm{pH}$-dependent autoxidation in aqueous media to produce a semiquinone intermediate and quinone product, ${ }^{25}$ which is thought to be further reacted into various compounds, such as catecholamine and indole. $^{22}$ Due to their reactive nature, the quinone derivatives produced can further undergo secondary reactions, such as Michael-type addition, Schiff base reaction, and Strecker degradation with thiols and amines or aryloxy radical coupling with the unoxidized catechol groups. ${ }^{26}$ While the versatile chemistry of catechol is undeniable, the polymerization mechanism, the deposition on the surface, and the structure of biophenols remain under debate despite great success in the fabrication of biophenol coatings. ${ }^{27}$

Although reports on polydopamine membranes for aqueous nanofiltration are abundant, ${ }^{18}$ their applications in OSN are relatively scarce albeit increasing. In order for the polydopamine-coated membranes to acquire stability in organic solvents, a further cross-linking step is usually required. ${ }^{23}$ However, disadvantages exist, such as additional membrane fabrication steps and environmental concerns. An alternative is dopamine codeposition with reactive agents, such as terephthaloyl chloride ${ }^{28}$ or amine-containing molecules, ${ }^{29}$ resulting in membranes that are stable in polar aprotic solvents. A possible explanation is that due to the presence of catecholquinone moieties and radical species, polydopamine exhibits intrinsic chemical reactivity. Through Michael-type addition reactions and/or Schiff-base formations, polydopamine can readily react with molecules with nucleophilic groups, such as thiolate $\left(-\mathrm{S}^{-}\right)$and amine $\left(-\mathrm{NH}_{2}\right)$, generating thiol-catechol or amine-catechol adducts. ${ }^{30}$ Membranes based on interpenetrating polymer networks of polybenzimidazole and polydopamine demonstrated excellent OSN performance. $^{31}$

Besides dopamine, other biophenols have been used for OSN applications. Interfacial polymerization of tannic acid, ${ }^{32}$ morin, ${ }^{33}$ and quercetin ${ }^{34}$ with terephthaloyl chloride; vanillic alcohol with trimesoyl chloride; ${ }^{35}$ and catechol with octaammonium polyhedral oligomeric silsesquioxane ${ }^{29}$ were reported to make thin-film composite OSN membranes. However, the use of an acyl chloride and annealing at high temperature $\left(85{ }^{\circ} \mathrm{C}\right)$ for complete interfacial polymerization makes it environmentally less benign.

Apart from autoxidation of dopamine at slightly alkaline $\mathrm{pH}$ ( $\mathrm{pH}$ 8.0-8.5), various oxidants (e.g., copper(II) sulfate and hydrogen peroxide, ${ }^{36}$ ammonium persulfate, ${ }^{37}$ sodium periodate, ${ }^{38}$ potassium permanganate, and iron(III)) were used to increase the coating speed and endow membranes with resistance toward harsh environments. ${ }^{39,40}$ In OSN applications, polydopamine deposition on a porous polyacrylonitrile support promoted by different oxidants was reported. ${ }^{41}$ However, previous publications are merely case studies on the influences of single biophenol coatings toward the filtration performance of single OSN membranes, which makes it difficult to make direct comparisons among different combinations.

Herein, we demonstrate the first systematic study where oxidant-promoted biophenol coating formation is used as a versatile, reproducible, and easily scalable method to fine-tune 
the pore size and, hence, separation performance of OSN membranes.

\section{EXPERIMENTAL SECTION}

Materials. PI was purchased from HP Polymer GmbH (Lenzing, Austria; product code, 58698-66-1). PAN was purchased from Goodfellow Cambridge Ltd. (Huntingdon, UK; product code, AN316020; $\left.M_{\mathrm{w}}, 85000 \mathrm{~g} \mathrm{~mol}^{-1}\right)$. PBI (26 wt \% in $N, N$ dimethylacetamide (DMAc)) was purchased from PBI Performance Products, Inc. (Charlotte, USA). Dopamine hydrochloride (99\%) and quercetin dihydrate (97\%) were purchased from Alfa Aesar. PSf (product code, 178912500; $M_{\mathrm{w}}, 60000 \mathrm{~g} \mathrm{~mol}^{-1}$ ), morin hydrate, heptane $(\geq 99 \%)$, acetonitrile $(\mathrm{MeCN}, \geq 99.9 \%)$, ethyl acetate (EtOAc, analytical reagent grade), acetone (analytical reagent grade), methanol $(\mathrm{MeOH}, \geq 99.8 \%)$, toluene (analytical reagent grade), dichloromethane (DCM, analytical reagent grade), and tetrahydrofuran (THF, analytical reagent grade) were purchased from Fisher Scientific. PVDF (product code, 427152; $M_{\mathrm{w}}, 180000 \mathrm{~g} \mathrm{~mol}^{-1} ; M_{\mathrm{n}}$, $71000 \mathrm{~g} \mathrm{~mol}^{-1}$ ), tannic acid (ACS reagent), eugenol (99\%), vanillyl alcohol (98\%), sodium periodate (analytical reagent grade), $N, N$ dimethylformamide (DMF, $\geq 99.8 \%$ ), N,N-dimethylacetamide (DMAc, $\geq 99.5 \%$ ), Cyrene (dihydrolevoglucosenone, 99\%), dimethyl carbonate (DMC, 99\%), propylene carbonate (PC, 99\%), and 2methyltetrahydrofuran (MeTHF, $\geq 99 \%$ ) were purchased from Sigma-Aldrich. Novatexx 2471 polypropylene nonwoven backing was purchased from Freudenberg Filtration Technologies (Crewe, UK). Polydimethylsiloxane (PDMS) membrane can be purchased from GMT Membrantechnik GmbH (Rheinfelden, Germany). Polystyrene markers $\left(236-1900 \mathrm{~g} \mathrm{~mol}^{-1}\right)$ for solute rejection evaluation were purchased from Agilent Technologies. Microscope slides were purchased from Thermo Scientific. All materials and solvents were used as received without further purification. All aqueous solutions were prepared using water with a resistivity of 18.2 $\mathrm{M} \Omega \mathrm{cm}$ at $25^{\circ} \mathrm{C}$ (Milli-Q).

Preparation of Pristine Polymer Membrane Supports. The PBI dope solution was prepared by diluting commercial PBI solutions in DMAc by mechanical stirring (6 h, $50 \mathrm{rpm}, \mathrm{RT})$ and then degassing under argon in an incubator shaker $\left(400 \mathrm{rpm}, 30^{\circ} \mathrm{C}, 12 \mathrm{~h}\right)$. Dope solutions of PSf, PVDF, and PAN were prepared in a similar fashion, by dissolving the respective polymer solids in DMAc under mechanical stirring $(50 \mathrm{rpm}, 6 \mathrm{~h})$ at 50,50 , and $65^{\circ} \mathrm{C}$, respectively, and then degassing as above. The dope solution of PI was prepared following the same procedure, except that DMF was used as the solvent because PI membranes cast from DMAc solution were found to break due to high brittleness during the phase inversion process. On the basis of the preliminary trials, the polymer concentrations for PI, PAN, PSf, PVDF, and PBI dope solutions were fixed at 22, 19, 24, 24 , and 19 wt $\%$, respectively, to obtain loose nanofiltration membranes. All the dope solutions contained $1.5 \mathrm{wt} \%$ lithium chloride as a pore-forming agent. Figure 2 illustrates the membrane

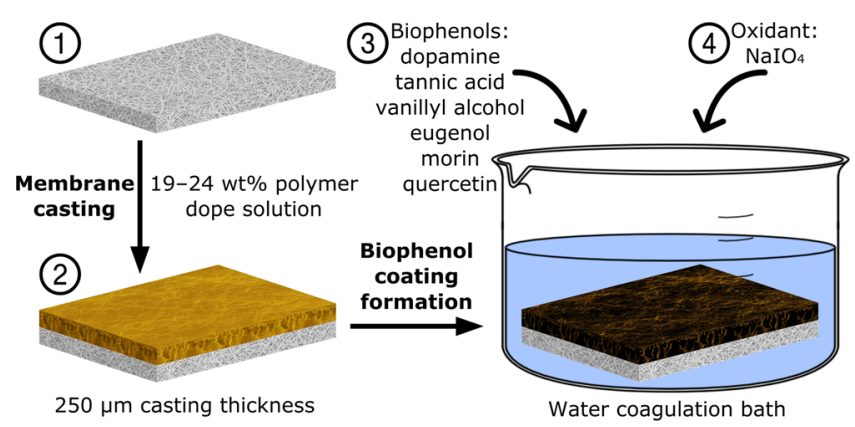

Figure 2. Schematic of the fabrication of biophenol-coated membranes. (1) Polypropylene nonwoven backing; (2) pristine polymer membrane on backing prepared by casting; biophenol-coated membrane prepared by soaking the pristine membrane in (3) biophenol solution followed by (4) oxidant addition. fabrication process. Membranes were formed as previously described by casting $250 \mu \mathrm{m}$ polymer films at $5 \mathrm{~cm} \mathrm{~s}^{-1}$ on a polypropylene support with a doctor blade, followed by phase inversion in deionized water $(15.0 \mathrm{M} \Omega \mathrm{cm})$ at $25{ }^{\circ} \mathrm{C} .{ }^{10}$ The membranes were cut into $9 \mathrm{~cm}$ diameter circles by hand and then stored in water containing $1 \mathrm{vol} \%$ $\mathrm{MeCN}$ before use.

Preparation of Biophenol-Coated Nanofiltration Membranes. In a typical procedure, dopamine-coated membranes were prepared by soaking the pristine polymer membrane discs having 9 $\mathrm{cm}$ diameter in $2 \mathrm{~g} \mathrm{~L}^{-1}$ aqueous dopamine solution for $24 \mathrm{~h}$, followed by the addition of $\mathrm{NaIO}_{4}$ to a final concentration of $5 \mathrm{mM}$ (pH 5) to promote polymerization for an additional $24 \mathrm{~h}$ (Figure 2). The coated membranes were rinsed with water and then stored in water containing 1 vol $\% \mathrm{MeCN}$. This two-step coating procedure was used to better control the coating formation process by allowing the infusion of the dopamine molecule into the porous structures of the membrane before its in situ polymerization under oxidative conditions. Coatings with other biophenols were performed in the same way to that of dopamine. Vanillyl alcohol, eugenol, morin, and quercetin were dissolved in a $1: 1(\mathrm{v}: \mathrm{v})$ water:ethanol mixture instead of water. The addition of $50 \%$ ethanol improves the dissolution of these biophenols, while 50\% water allows for the dissolution of $\mathrm{NaIO}_{4} \cdot{ }^{33}$ A list of all the membranes prepared in this work can be found in Table 1. Standalone polyphenols were also synthesized under the same conditions as the membrane coating in order for a direct comparison of attenuated total reflection Fourier-transform infrared spectroscopy (ATR-FTIR) and thermogravimetric analysis (TGA) between coated membranes and the coating material.

Membrane Characterization. ATR-FTIR spectra of membranes were obtained using a Bruker Alpha-P. The samples were mounted on a zinc-selenium/diamond plate, and the measurements were run in air. The average of 16 scans at a resolution of $4 \mathrm{~cm}^{-1}$ was used to generate the spectra. Raman spectra were recorded using an InVia spectrometer (Renishaw) with a $633 \mathrm{~nm}$ laser. The membrane surface and cross-sectional microstructure were studied by scanning electron microscopy (SEM) using a Zeiss Ultra55 with an in-lens detector and a field emission gun with the accelerating voltage set to $3 \mathrm{kV}$. Dried membranes were stuck on the sample holder by conductive carbon tab. A sputter coater (Quorum Q150TES) was used to generate a 6 $\mathrm{nm}$ gold/palladium coating on samples in order to make them conductive. A MultiMode 8 with a Bruker TESPA-V2 probe was used to obtain atomic force microscopy (AFM) images. TappingMode in air was used. Dried membranes were stuck on a glass slide using double-sided tape. The roughness of samples were analyzed using the NanoScope Analysis software on an $2 \mu \mathrm{m} \times 2 \mu \mathrm{m}$ area, and three membranes of each type were tested and averaged. A TA Instruments Q500 was used to determine the TGA results of samples in an $\mathrm{N}_{2}$ atmosphere with the ramp rate of $20{ }^{\circ} \mathrm{C} \mathrm{min}^{-1}$. Water contact angles of membranes were obtained by using Krüss DSA100. Then, $1.5 \mu \mathrm{L}$ of deionized water was injected on the membrane surface, and the contact angle was obtained by using the sessile drop fitting function in Drop Shape Analysis (version 1.91.0.2). The solvent stability of membranes were tested by soaking membranes in eight common organic solvents, heptane, EtOAc, acetone, $\mathrm{MeOH}$, toluene, DCM, THF, and DMF, and four green solvents, DMC, PC, MeTHF, and Cyrene (dihydrolevoglucosenone). Membranes that are stable in THF and Cyrene were further tested for their swellings in both solvents. Swelling of a membrane is quantified by measuring its original thickness in water and its swelling thickness after being soaked in THF or Cyrene for $24 \mathrm{~h}$. Thickness of the membrane was measured by using a digital caliper (Site UK Limited) with $10 \mu \mathrm{m}$ accuracy. Each membrane sample was measured 10 times. The swelling ratio is reported as the relative change in the membrane final thickness in organic solvents as compared to their original thickness in water.

Separation Performance of Nanofiltration Membranes. Membrane separation performance was examined in a stainless steel cross-flow filtration apparatus at $10 \mathrm{bar}$ (Figure 3). All the tests were carried out for at least 1 week. A long-term aging test of $\mathrm{M}_{\mathrm{PVDF}}^{\mathrm{d}}$ in MeTHF was carried out for 3 months. Two independent duplicates of 
Table 1. Abbreviations of Membranes ${ }^{a}$

\begin{tabular}{|c|c|c|c|c|c|c|c|}
\hline coatings & no coating & dopamine & tannic & vanillyl alcohol & eugenol & morin & quercetin \\
\hline PI & $\mathrm{M}_{\mathrm{PI}}$ & $\mathrm{M}_{\mathrm{PI}}^{\mathrm{d}}$ & $\mathrm{M}_{\mathrm{PI}}^{\mathrm{t}}$ & $\mathrm{M}_{\mathrm{PI}}^{\mathrm{v}}$ & $\mathrm{M}_{\mathrm{PI}}^{\mathrm{e}}$ & $\mathrm{M}_{\mathrm{PI}}^{\mathrm{m}}$ & $\mathrm{M}_{\mathrm{PI}}^{\mathrm{q}}$ \\
\hline PAN & $\mathrm{M}_{\mathrm{PAN}}$ & $\mathrm{M}_{\mathrm{PAN}}^{\mathrm{d}}$ & $\mathrm{M}_{\mathrm{PAN}}^{\mathrm{t}}$ & $\mathrm{M}_{\mathrm{PAN}}^{\mathrm{v}}$ & $\mathrm{M}_{\mathrm{PAN}}^{\mathrm{e}}$ & $\mathrm{M}_{\mathrm{PAN}}^{\mathrm{m}}$ & $\mathrm{M}_{\mathrm{PAN}}^{\mathrm{q}}$ \\
\hline PSf & $\mathrm{M}_{\mathrm{PSf}}$ & $\mathrm{M}_{\mathrm{PSf}}^{\mathrm{d}}$ & $\mathrm{M}_{\mathrm{PSf}}^{\mathrm{t}}$ & $\mathrm{M}_{\mathrm{PSf}}^{\mathrm{v}}$ & $\mathrm{M}_{\mathrm{PSf}}^{\mathrm{e}}$ & $\mathrm{M}_{\mathrm{PSf}}^{\mathrm{m}}$ & $\mathrm{M}_{\mathrm{PSf}}^{\mathrm{q}}$ \\
\hline PVDF & $\mathrm{M}_{\mathrm{PVDF}}$ & $\mathrm{M}_{\mathrm{PVDF}}^{\mathrm{d}}$ & $\mathrm{M}_{\mathrm{PVDF}}^{\mathrm{t}}$ & $\mathrm{M}_{\mathrm{PVDF}}^{\mathrm{v}}$ & $\mathrm{M}_{\mathrm{PVDF}}^{\mathrm{e}}$ & $\mathrm{M}_{\mathrm{PVDF}}^{\mathrm{m}}$ & $\mathrm{M}_{\mathrm{PVDF}}^{\mathrm{q}}$ \\
\hline PBI & $\mathrm{M}_{\mathrm{PBI}}$ & $\mathrm{M}_{\mathrm{PBI}}^{\mathrm{d}}$ & $\mathrm{M}_{\mathrm{PBI}}^{\mathrm{t}}$ & $\mathrm{M}_{\mathrm{PBI}}^{\mathrm{v}}$ & $\mathrm{M}_{\mathrm{PBI}}^{\mathrm{e}}$ & $\mathrm{M}_{\mathrm{PBI}}^{\mathrm{m}}$ & $\mathrm{M}_{\mathrm{PBI}}^{\mathrm{q}}$ \\
\hline PDMS & $\mathrm{M}_{\mathrm{PDMS}}$ & $\mathrm{M}_{\mathrm{PDMS}}^{\mathrm{d}}$ & $\mathrm{M}_{\mathrm{PDMS}}^{\mathrm{t}}$ & $\mathrm{M}_{\mathrm{PDMS}}^{\mathrm{v}}$ & $\mathrm{M}_{\mathrm{PDMS}}^{\mathrm{e}}$ & $\mathrm{M}_{\mathrm{PDMS}}^{\mathrm{m}}$ & $\mathrm{M}_{\mathrm{PDMS}}^{\mathrm{q}}$ \\
\hline
\end{tabular}

${ }^{a_{T}}$ The subscript refers to the membrane material and the superscript refers to the biophenol coating, where present.

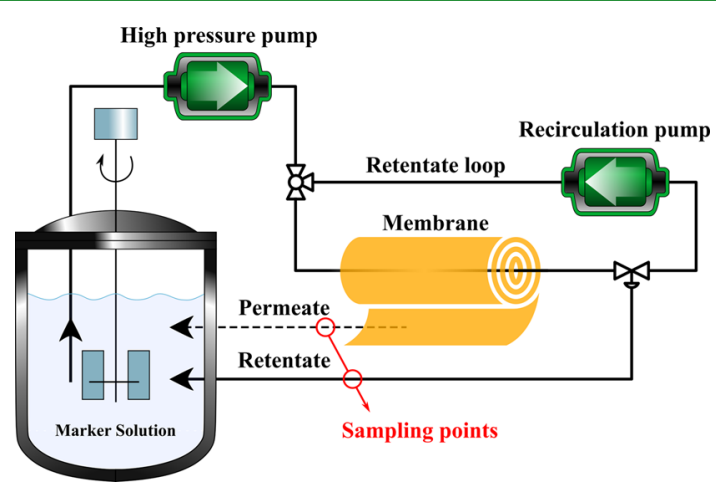

Figure 3. Schematic process configuration for membrane screening, i.e., determination of solvent permeance and solute rejection.

each membrane were prepared, and each membrane prepared was tested three times; the reported results are the mean values of these measurements. The effective area $(A)$ of each membrane was $52.8 \mathrm{~cm}^{2}$. Equation 1 was used to calculate the permeance.

$$
\text { Permeance }=\frac{J}{\Delta P}=\frac{V}{\Delta P A t}
$$

In the equation, the solvent flux through the membrane $(J)$ was divided by the transmembrane pressure $(\Delta P)$ to obtain permeance.
The flux was the volume of solvent $(V)$ that permeates through the membrane for a given membrane area $(A)$ in a given time $(t)$. Rejection values were determined by using a standard containing $1 \mathrm{~g}$ $\mathrm{L}^{-1}$ of PS580 and PS1300 polystyrene markers and $0.1 \mathrm{~g} \mathrm{~L}^{-1}$ of methylstyrene dimer $\left(236 \mathrm{~g} \mathrm{~mol}^{-1}\right)$ in acetone, as previously describe. $^{10,42}$ As defined in eq 2 , the measured concentration of solutes in the permeate $\left(C_{\mathrm{p}}\right)$ and the feed $\left(C_{\mathrm{f}}\right)$ was used to determine the rejection $(R)$.

$$
\text { Rejection }(\%)=\left(1-\frac{C_{\mathrm{p}}}{C_{\mathrm{f}}}\right) \times 100 \%
$$

Molecular weight cutoff (MWCO) is defined as the lowest molecular weight solute in which $90 \%$ of it is retained by the membrane, and it was estimated from the rejections curve in this study by linear interpolation. In order to compare the effects of different biophenol coatings toward the permeance and MWCO for different polymer supports, relative changes are calculated by using eqs 3 and 4.

$$
\begin{aligned}
& \text { Relative change in permeance (\%) } \\
& =\left(1-\frac{\text { Permeance }_{\text {coated }}}{\text { Permeance }_{\text {pristine }}}\right) \times 100 \%
\end{aligned}
$$
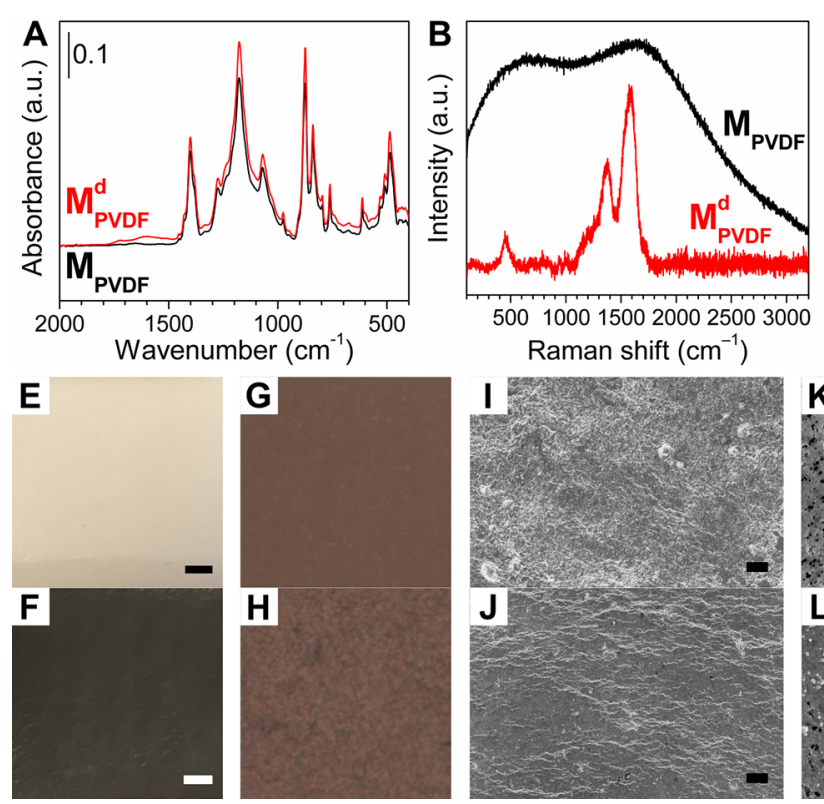
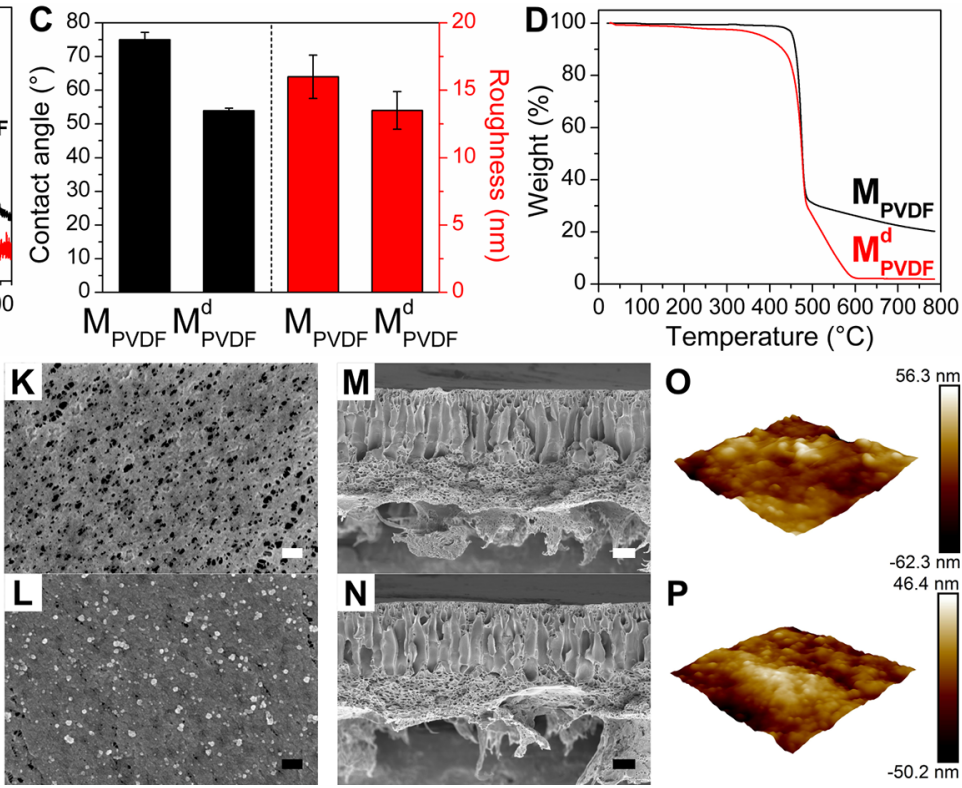

Figure 4. Physicochemical characterizations of representative membranes $\mathrm{M}_{\mathrm{PVDF}}$ and $\mathrm{M}_{\mathrm{PVDF}}^{\mathrm{d}}$. (A) ATR-FTIR spectra. (B) Raman spectra. (C) Water contact angle and surface roughness (root-mean-square). (D) TGA. (E, F) Appearance (scale bar $=2 \mathrm{~mm}$ ). (G, H) Visible-light microscope images (reflection mode, frame size $=100 \mu \mathrm{m} \times 100 \mu \mathrm{m})$. (I, J) Lower-magnification SEM images of membrane surface $($ scale bar $=20 \mu \mathrm{m})$. $(\mathrm{K}$, L) Higher-magnification SEM images of membrane surface $($ scale bar $=200 \mathrm{~nm}) .(\mathrm{M}, \mathrm{N})$ SEM images of membrane cross sections $($ scale bar $=10$ $\mu \mathrm{m})$. (O, P) AFM height images of membranes (scan size $=2 \mu \mathrm{m} \times 2 \mu \mathrm{m})$. 

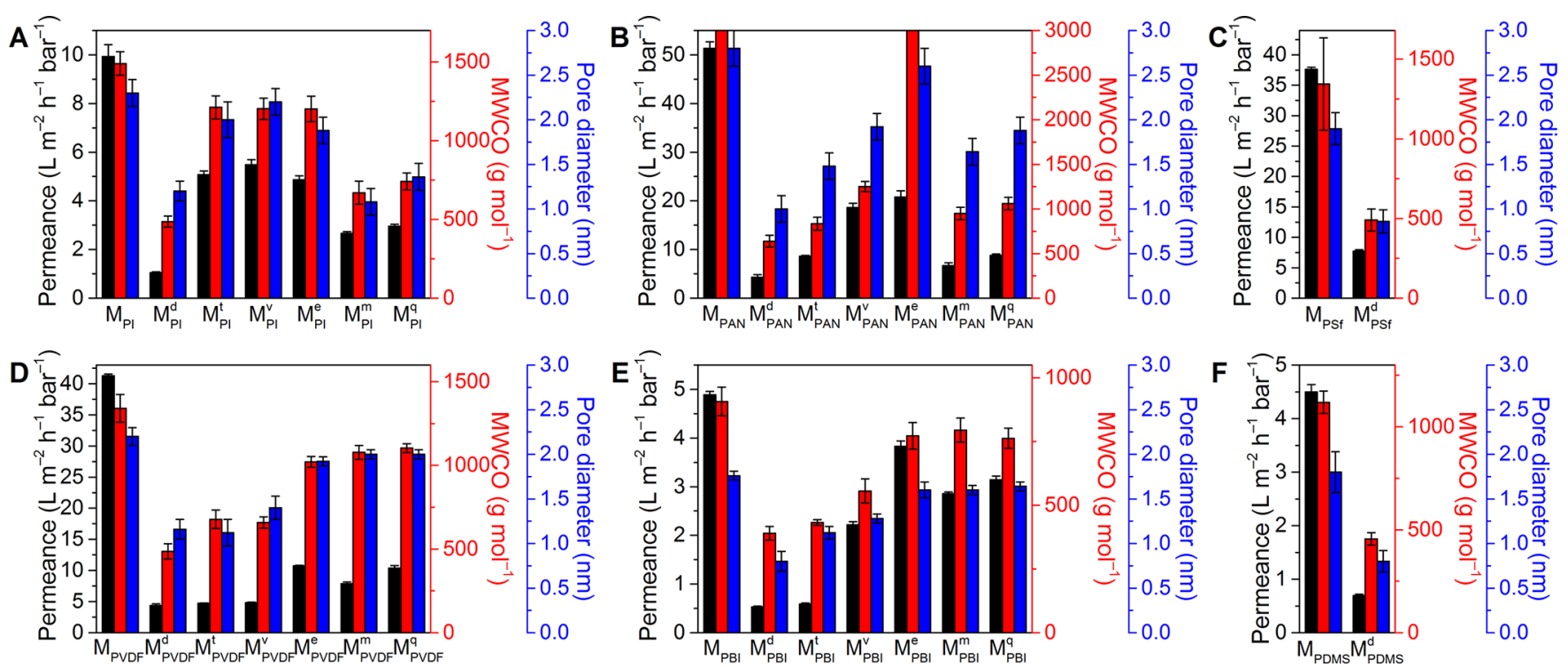

Figure 5. Permeance, cutoff and pore size of (A) PI, (B) PAN, (C) PSf, (D) PVDF, (E) PBI, and (F) PDMS membranes in acetone. All the data are average values of two pieces of independently prepared membranes at two data collection points at $24 \mathrm{~h}$ and 7 days. Only the dopamine coating was found to be stable on PSf (C) and PDMS (F) membranes in acetone. The standard error of MWCO of PSf (C) is large due to the increase of MWCO during the one-week testing period (see Figure S24C). A transposed version of the figure with the same data where single coatings are compared within single panels can be found in the SI.

Relative change in rejection (\%)

$$
=\left(1-\frac{\text { Rejection }_{\text {coated }}}{\text { Rejection }_{\text {pristine }}}\right) \times 100 \%
$$

\section{RESULTS AND DISCUSSION}

As a representative example, the physicochemical characterization of the membranes is exemplified on $M_{P V D F}$ and $M_{P V D F}^{d}$ membranes in Figure 4. Owing to the large number of membranes prepared in this study, the other membranes can be found in the SI. The formation of the biophenol coatings can be easily observed from the change in the color of the membrane surface (Figures 4E,F and S6). The polymerized biophenols are black, and consequently, the coating procedure darkens the membrane surface to different degrees depending on the type of polymers and biophenols.

ATR-FTIR results (Figures 4A, S3, and S4) confirmed the formation of different biophenol coatings on all of the six polymer membranes. Compared with pristine membranes, biophenol-coated membranes showed increased absorbance across the whole spectra and especially on existing polymer peaks (Table S3), which can be attributed to the deposition of an organic film. ${ }^{43}$ Significantly increased phenol O-H stretch at 3200 to $3400 \mathrm{~cm}^{-1}$ for all the biophenol-coated membranes suggested that hydroxyl groups are abundant after polymerization. $^{44}$

Membrane morphologies were characterized by SEM and AFM. SEM images (Figures 4I-N and S8-S10) showed the typical morphology of membranes prepared by phase inversion. However, SEM was hardly able to distinguish biophenol-coated membranes from their pristine counterparts (Figures 4I,J and S8), due to (i) similar secondary electron yields compared to the underlying membranes and (ii) the small thickness of the coatings. In the higher magnification SEM plan view images (Figures 4K,L and S9), a small number of particles can be observed from biophenol-coated mem- branes, which might be from the precipitation route of biophenol polymerization, acting in competition with thin film formation. ${ }^{45}$ In the SEM cross-sectional images (Figures 4M,N and S10), no apparent interphase can be observed on the top of membranes, implying good compatibility between the coatings and the membranes. ${ }^{46}$ AFM height images (Figures $4 \mathrm{O}, \mathrm{P}$ and S11) provided similar surface morphology information with that of higher magnification SEM surface images. There is no clear trend in the influence of biophenolcoatings toward the surface roughness of membranes (Table S4). Biophenol coatings increased the hydrophilicity of membranes in various degrees $(3-45 \%$ corresponding to $2-$ $34^{\circ}$, Figure S5), which is from the abundant hydroxyl groups in the biophenol coatings. ${ }^{47}$

All the membranes were tested by Raman spectroscopy. However, spectra can only be obtained from dopamine-coated membranes (Figures 4B and S12); all the other membranes (including pristine ones) showed broad fluorescence across the whole spectra, and no peaks could be observed. The fluorescence is likely to be from the chemistry of the membranes. Note, that the polydopamine coating can mask the Raman fluorescence of the substrate. Although Raman spectroscopy has been used to verify the existence of polydopamine, ${ }^{48}$ to the best of our knowledge, it is the first time that Raman mapping is used to verify the homogeneity (with $1 \mu \mathrm{m}$ lateral resolution) of dopamine coatings on 6 different polymer membrane supports (Figure S13).

The majority of the membranes were stable in all 12 solvents tested, except for polar aprotic Cyrene and DMF (Table S7, Figures S32-S67). However, all of the PSf membranes (pristine and biophenol-coated) dissolved in toluene, DCM, MeTHF, and THF, which reveals that PSf (even with biophenol coatings) is not a robust material for OSN. Furthermore, the $\mathrm{M}_{\mathrm{PDMS}}^{\mathrm{d}}$ membrane from the PDMS set was found to be more stable than the rest. The dissolution results illustrate that the solvent stability of biophenol-coated 

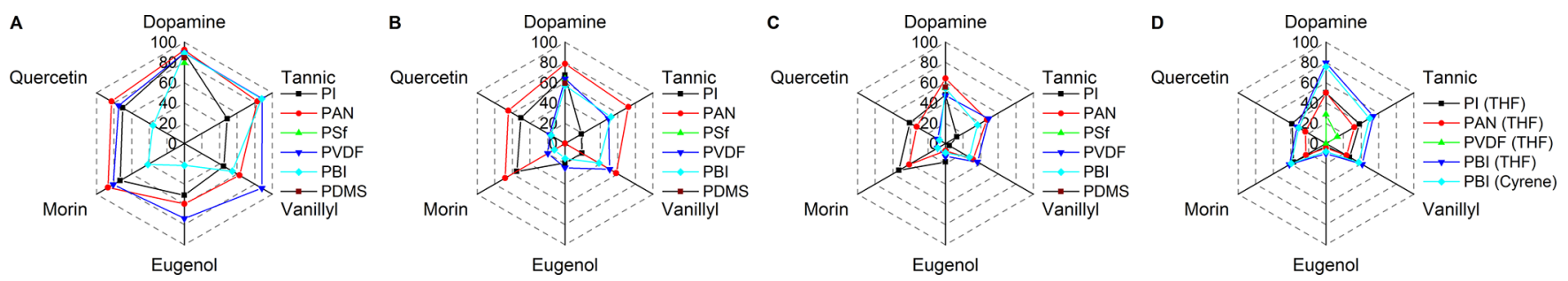

Figure 6. Relative change in (A) permeance, (B) MWCO, (C) pore size, and (D) swelling ratio of membranes (radar chart).

membranes is substrate-dependent. Nevertheless, the majority of the coating-substrate combinations were stable.

The stability of membranes were also tested in polar aprotic solvents Cyrene and DMF. The results showed that Cyrene dissolved all the membranes, except for dopamine-coated PAN, dopamine-coated PVDF, all of the PBI membranes, and all of the PDMS membranes. However, in contrast to PBI, where the pristine PBI and all the biophenol-coated membranes are stable in Cyrene, all the coatings, except for dopamine on PDMS, are dissolved by Cyrene. PBI is a robust material which enables biophenol coatings that can withstand polar aprotic solvent Cyrene, and dopamine coating can endow stability toward several polymer materials against Cyrene. Consequently, Cyrene has been identified as a green alternative for conventional polar aprotic solvents, which does not dissolve PBI and certain biophenol/polymer pairs contrary to the expectations. These findings can open new membrane processing possibilities in polar aprotic solvents.

The permeance of all the acetone-stable membranes are shown in Figure 5. Compared with pristine membranes, all the biophenol-coated membranes showed decreased permeance (by $22-92 \%$ ), which could be explained by the reduced size of pores in the membrane top layer due to biophenol deposition (see Section 5 in the SI for pore size estimation). Among all the biophenol coatings, dopamine leads to the greatest permeance decrease for all of the membrane materials. The influence is as high as 1 order of magnitude for PI, PAN, and PBI. Moreover, all the membranes showed relatively stable permeance (relative change within 5\%) during the one-week continuous filtration experiment, which verifies their robustness (Figure S16).

The rejection curves of all of the membranes toward PS markers are shown in Figures S17-S23, which were used to determine the MWCO and estimate the pore size of the membranes (Figure 5). The results show that all the biophenol coatings lead to decreased MWCO (by 12-79\%) compared to the pristine membranes, which is consistent with the observations on the permeance (Figure 5).

In order to facilitate a straightforward comparison of separation performance among different membranes, relative changes in permeance, MWCO, and pore size were calculated and are shown in Figure 6. As the MWCO of $\mathrm{M}_{\text {PAN }}$ and $\mathrm{M}_{\mathrm{PAN}}^{\mathrm{e}}$ exceed $2000 \mathrm{~g} \mathrm{~mol}^{-1}$ and no accurate numbers could be estimated from Figure $S 19,3000 \mathrm{~g} \mathrm{~mol}^{-1}$ was arbitrarily set as the MWCO for both $\mathrm{M}_{\mathrm{PAN}}$ and $\mathrm{M}_{\mathrm{PAN}}^{\mathrm{e}}$, which is not ideal (resulting in the no change in MWCO of $\mathrm{M}_{\mathrm{PAN}}^{\mathrm{e}}$ in Figure 6B) but necessary in order to complete the comparison. The results showed that changes in permeance, MWCO, and pore diameter of coated membranes are positively correlated (Figure 7). This phenomenon can be explained by the decrease in pore size due to the deposition of the biophenols, which in turn leads to a decrease in both solvent permeance

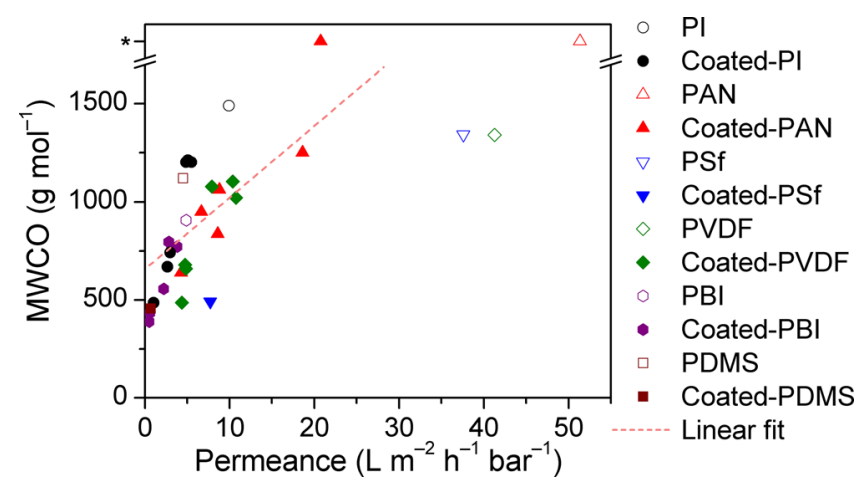

Figure 7. Permeance versus MWCO for all 32 membranes tested in acetone. The asterisk indicates that the MWCO is higher than the tested nanofiltration range (see Figure S19). Pearson correlation coefficient $(R), 0.7299$.

and MWCO. For each biophenol-coated membrane, as compared to its pristine membrane, the relative change in permeance is larger than that in MWCO. Consequently, to obtain tighter membranes through biophenol coating formation, the permeance needs to be compromised. ${ }^{49}$ Nevertheless, the capability of biophenol coatings on the fine-tuning of membrane separation performance has been unambiguously confirmed (Figure 6).

Membranes that are stable in THF and Cyrene (polar aprotic solvents) were also characterized by their swelling in both solvents (Figure 6D), which are well-known to severely swell polymer membranes. The swelling ratio results (Figure S68) show that different membranes swell in different degrees in THF and Cyrene, but the trends are similar. Among the pristine membranes, PBI swells most (32\%), while PDMS swells least (3\%). Biophenol coatings can alleviate membrane swellings. Among them, dopamine has the largest effect, while eugenol has the smallest effect, which could be observed more apparently in Figure 6D. The swelling ratio of PBI membrane decreased nearly 5 times after dopamine is coated on it. The alleviation of membrane swelling by biophenol coatings is thought to be from a combination of enhanced interactions in polymer networks (Figure 1) and slightly increased membrane hydrophilicity (Figure S5).

Similar to Figure 5, $\mathrm{M}_{\mathrm{PVDF}}$ and $\mathrm{M}_{\mathrm{PVDF}}^{\mathrm{d}}$ were selected to test the membrane performance in an additional four green organic solvents with varying polarity, MeTHF, toluene, EtOAc, and heptane (Figure 8A). The results show that $\mathrm{M}_{\mathrm{PVDF}}^{\mathrm{d}}$ has lower permeance and lower MWCO than $\mathrm{M}_{\mathrm{PVDF}}$ in all of the solvents. There is a positive correlation between permeance and the solubility parameter of the solvents for $\mathrm{M}_{\mathrm{PVDF}}$ (Figure S31A). This observation is consistent with a previous report that similar Hansen solubility parameters between a solvent and a membrane suggest a stronger solvent-membrane interaction, leading to higher permeance. ${ }^{50}$ In contrast, $\mathrm{M}_{\mathrm{PVDF}}^{\mathrm{d}}$ 

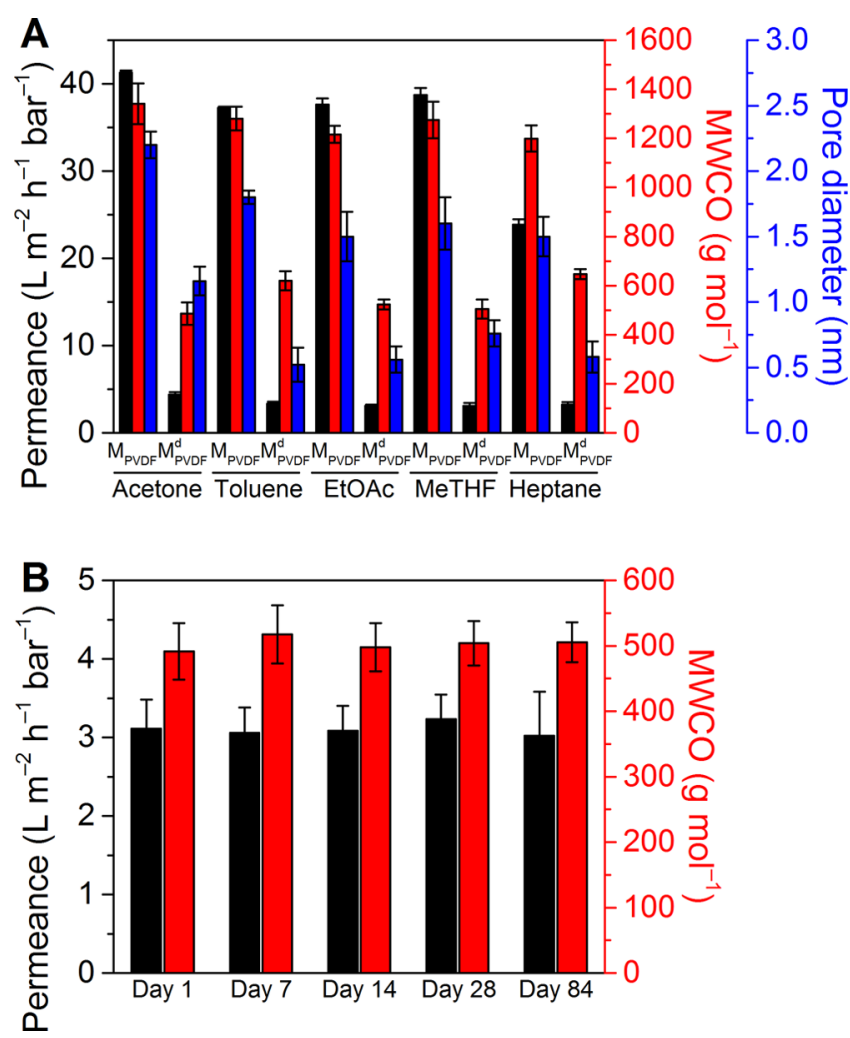

Figure 8. (A) Permeance, cutoff, and pore size of $\mathrm{M}_{\mathrm{PVDF}}$ and $\mathrm{M}_{\mathrm{PVDF}}^{\mathrm{d}}$ in different organic solvents. The data as a function of HSP are presented in Figure S31. (B) Long-term permeance and molecular weight cutoff of $\mathrm{M}_{\mathrm{PVDF}}^{\mathrm{d}}$ in MeTHF.

has a similar permeance irrespective of solvent polarity. The MWCO and estimated pore size remained quasi constant for both membranes in all solvents (Figure 8A). These results demonstrate that biophenol coatings could result in more robust membranes, which perform the same irrespective of solvent selection. A long-term aging test of $\mathrm{M}_{\mathrm{PVDF}}^{\mathrm{d}}$ in MeTHF (Figure $8 \mathrm{~B}$ ) verifies that membrane separation performance is stable for at least 3 months.

\section{CONCLUSIONS}

This systematic study verifies the versatile method that utilizes low-cost biophenol coatings for fine-tuning the separation performance of polymer OSN membranes, and can be more generally adapted to phenolic coatings on polymer surfaces. The majority of biophenol coatings are stable in various organic solvents in the 1.9-20.7 dielectric constant range. All the biophenol coatings lead to decreased permeance of solvent $(22-92 \%)$ and increased rejection of solutes (12-79\%). In addition, biophenol coatings can decrease the swelling by up to $80 \%$, resulting in more stable membranes in organic solvents. Among the tannic acid, vanillyl alcohol, eugenol, morin, quercetin, and dopamine coatings, the latter has the most profound effect on the membrane performance. Moreover, dopamine coating endows PAN and PVDF membranes with solvent resistance toward the green polar aprotic solvent Cyrene. Dopamine-coated PVDF membrane demonstrated stable permeance and rejection in MeTHF for over 3 months of operation. Apart from membranes, the results could be further exploited in the fields of food packaging and biomedical products.

\section{ASSOCIATED CONTENT}

\section{Supporting Information}

The Supporting Information is available free of charge on the ACS Publications website at DOI: 10.1021/acsapm.8b00161.

Chemical structures of membrane polymers and biophenols, preparation of standalone polyphenols, physicochemical characterization of membranes, membrane performance in nanofiltration, solvent permeance and MWCO correlation analysis, and membrane dissolution test. (PDF)

\section{AUTHOR INFORMATION}

\section{Corresponding Authors}

*E-mail: christopher.blanford@manchester.ac.uk

*E-mail: gyorgy.szekely@manchester.ac.uk, www.szekelygroup. com..

ORCID $\odot$

Fan Fei: 0000-0003-0548-5775

Hai Anh Le Phuong: 0000-0003-1380-4799

Christopher F. Blanford: 0000-0002-0112-7818

Gyorgy Szekely: 0000-0001-9658-2452

\section{Present Address}

(G.S.): Advanced Membranes and Porous Materials (AMPM) Center, King Abdullah University of Science and Technology (KAUST), Thuwal, 23955-6900, Saudi Arabia

\section{Notes}

The authors declare no competing financial interest. In accordance with the University of Manchester's guidelines, the data are openly available from Mendeley Data (https:// data.mendeley.com/datasets/8w8xk6htcg/1).

\section{ACKNOWLEDGMENTS}

The authors give thanks for the experimental support from Abdulaziz Alammar (TGA) and the scientific discussions with Levente Cseri. This work was supported by the UK's Biotechnology and Biological Sciences Research Council (BBSRC) and Engineering and Physical Sciences Research Council (EPSRC) through an award from the BioProNET Network in Industrial Biotechnology and Bioenergy (BB/ L013770/1). F.F. acknowledges the financial support from his family for his doctoral studies. H.A.L.P. acknowledges the financial support from EPSRC Doctoral Training Award.

\section{ABBREVIATIONS}

MWCO, molecular weight cutoff; OSN, organic solvent nanofiltration; PI, polyimide; PAN, polyacrylonitrile; PSf, polysulfone; PVDF, polyvinylidene difluoride; PBI, polybenzimidazole; PDMS, polydimethylsiloxane; DMAc, $N, N$-dimethylacetamide; DMF, N,N-dimethylformamide; EtOAc, ethyl acetate; $\mathrm{MeOH}$, methanol; $\mathrm{MeCN}$, acetonitrile; DMC, dimethyl carbonate; PC, propylene carbonate; DCM, dichloromethane; THF, tetrahydrofuran; MeTHF, 2-methyltetrahydrofuran

\section{REFERENCES}

(1) Marchetti, P.; Jimenez Solomon, M. F.; Szekely, G.; Livingston, A. G. Molecular Separation with Organic Solvent Nanofiltration: A Critical Review. Chem. Rev. 2014, 114, 10735-10806.

(2) Szekely, G.; Jimenez-Solomon, M. F.; Marchetti, P.; Kim, J. F.; Livingston, A. G. Sustainability Assessment of Organic Solvent 
Nanofiltration: From Fabrication to Application. Green Chem. 2014, 16, 4440-4473.

(3) Szekely, G.; Gil, M.; Sellergren, B.; Heggie, W.; Ferreira, F. C. Environmental and Economic Analysis for Selection and Engineering Sustainable API Degenotoxification Processes. Green Chem. 2013, 15, 210-225.

(4) Guillen, G. R.; Pan, Y.; Li, M.; Hoek, E. M. Preparation and Characterization of Membranes Formed by Nonsolvent Induced Phase Separation: A Review. Ind. Eng. Chem. Res. 2011, 50, 37983817.

(5) Hołda, A. K.; Vankelecom, I. F. Understanding and Guiding the Phase Inversion Process for Synthesis of Solvent Resistant Nanofiltration Membranes. J. Appl. Polym. Sci. 2015, 132, 42130.

(6) Soroko, I.; Lopes, M. P.; Livingston, A. The Effect of Membrane Formation Parameters on Performance of Polyimide Membranes for Organic Solvent Nanofiltration (OSN): Part A. Effect of Polymer/ Solvent/Non-Solvent System Choice. J. Membr. Sci. 2011, 381, 152162.

(7) Kim, I.-C.; Yun, H.-G.; Lee, K.-H. Preparation of Asymmetric Polyacrylonitrile Membrane with Small Pore Size by Phase Inversion and Post-Treatment Process. J. Membr. Sci. 2002, 199, 75-84.

(8) Hołda, A. K.; Aernouts, B.; Saeys, W.; Vankelecom, I. F. Study of Polymer Concentration and Evaporation Time as Phase Inversion Parameters for Polysulfone-Based SRNF Membranes. J. Membr. Sci. 2013, 442, 196-205.

(9) Van Goethem, C.; Mertens, M.; Vankelecom, I. F. J. Crosslinked PVDF Membranes for Aqueous and Extreme pH Nanofiltration. J. Membr. Sci. 2019, 572, 489-495.

(10) Fei, F.; Cseri, L.; Szekely, G.; Blanford, C. F. Robust Covalently Cross-linked Polybenzimidazole/Graphene Oxide Membranes for High-Flux Organic Solvent Nanofiltration. ACS Appl. Mater. Interfaces 2018, 10, 16140-16147.

(11) da Silva Burgal, J.; Peeva, L.; Livingston, A. Negligible Ageing in Poly(ether-ether-ketone) Membranes Widens Application Range for Solvent Processing. J. Membr. Sci. 2017, 525, 48-56.

(12) Pulido, B.; Chisca, S.; Nunes, S. P. Solvent and Thermal Resistant Ultrafiltration Membranes from Alkyne-Functionalized High-Performance Polymers. J. Membr. Sci. 2018, 564, 361-371.

(13) Yuan, S.; Wang, J.; Li, X.; Zhu, J.; Volodine, A.; Wang, X.; Yang, J.; Van Puyvelde, P.; Van der Bruggen, B. New Promising Polymer for Organic Solvent Nanofiltration: Oxidized Poly (Arylene Sulfide Sulfone). J. Membr. Sci. 2018, 549, 438-445.

(14) Kim, J. H.; Cook, M.; Park, S. H.; Moon, S. J.; Kim, J. F.; Livingston, A. G.; Lee, Y. M. A Compact and Scalable Fabrication Method for Robust Thin Film Composite Membranes. Green Chem. 2018, 20, 1887-1898.

(15) Amirilargani, M.; Sadrzadeh, M.; Sudhölter, E.; de Smet, L. Surface Modification Methods of Organic Solvent Nanofiltration Membranes. Chem. Eng. J. 2016, 289, 562-582.

(16) Liu, Y.; Ai, K.; Lu, L. Polydopamine and Its Derivative Materials: Synthesis and Promising Applications in Energy, Environmental, and Biomedical Fields. Chem. Rev. 2014, 114, 5057-5115.

(17) Barclay, T. G.; Hegab, H. M.; Clarke, S. R.; Ginic-Markovic, M. Versatile Surface Modification Using Polydopamine and Related Polycatecholamines: Chemistry, Structure, and Applications. Adv. Mater. Interfaces 2017, 4, 1601192.

(18) Yang, H. C.; Waldman, R. Z.; Wu, M. B.; Hou, J.; Chen, L.; Darling, S. B.; Xu, Z. K. Dopamine: Just the Right Medicine for Membranes. Adv. Funct. Mater. 2018, 28, 1705327.

(19) Sileika, T. S.; Barrett, D. G.; Zhang, R.; Lau, K. H. A.; Messersmith, P. B. Colorless Multifunctional Coatings Inspired by Polyphenols Found in Tea, Chocolate, and Wine. Angew. Chem., Int. Ed. 2013, 52, 10766-10770.

(20) Barrett, D. G.; Sileika, T. S.; Messersmith, P. B. Molecular Diversity in Phenolic and Polyphenolic Precursors of Tannin-Inspired Nanocoatings. Chem. Commun. 2014, 50, 7265-7268.

(21) Hong, S.; Yeom, J.; Song, I. T.; Kang, S. M.; Lee, H.; Lee, H. Pyrogallol 2-Aminoethane: A Plant Flavonoid-Inspired Molecule for
Material-Independent Surface Chemistry. Adv. Mater. Interfaces 2014, $1,1400113$.

(22) Ryu, J. H.; Messersmith, P. B.; Lee, H. Polydopamine Surface Chemistry: A Decade of Discovery. ACS Appl. Mater. Interfaces 2018, 10, 7523-7540.

(23) Xu, Y.; You, F.; Sun, H.; Shao, L. Realizing Mussel-Inspired Polydopamine Selective Layer with Strong Solvent Resistance in Nanofiltration toward Sustainable Reclamation. ACS Sustainable Chem. Eng. 2017, 5, 5520-5528.

(24) Lee, H.; Dellatore, S. M.; Miller, W. M.; Messersmith, P. B. Mussel-Inspired Surface Chemistry for Multifunctional Coatings. Science 2007, 318, 426-430.

(25) Della Vecchia, N. F.; Avolio, R.; Alfê, M.; Errico, M. E.; Napolitano, A.; d'Ischia, M. Building-Block Diversity in Polydopamine Underpins a Multifunctional Eumelanin-Type Platform Tunable Through a Quinone Control Point. Adv. Funct. Mater. 2013, 23, 1331-1340.

(26) Maier, G. P.; Butler, A. Siderophores and Mussel Foot Proteins: The Role of Catechol, Cations, and Metal Coordination in Surface Adhesion. JBIC, J. Biol. Inorg. Chem. 2017, 22, 739-749.

(27) Hong, S.; Na, Y. S.; Choi, S.; Song, I. T.; Kim, W. Y.; Lee, H. Non-Covalent Self-Assembly and Covalent Polymerization CoContribute to Polydopamine Formation. Adv. Funct. Mater. 2012, 22, 4711-4717.

(28) Pérez-Manríquez, L.; Behzad, A. R.; Peinemann, K.-V. Sub-6 nm Thin Cross-Linked Dopamine Films with High Pressure Stability for Organic Solvent Nanofiltration. Macromol. Mater. Eng. 2016, 301, $1437-1442$.

(29) Xu, Y. C.; Tang, Y. P.; Liu, L. F.; Guo, Z. H.; Shao, L. Nanocomposite Organic Solvent Nanofiltration Membranes by a Highly-Efficient Mussel-Inspired Co-Deposition Strategy. J. Membr. Sci. 2017, 526, 32-42.

(30) Huang, N.; Zhang, S.; Yang, L.; Liu, M.; Li, H.; Zhang, Y.; Yao, S. Multifunctional Electrochemical Platforms Based on the Michael Addition/Schiff Base Reaction of Polydopamine Modified Reduced Graphene Oxide: Construction and Application. ACS Appl. Mater. Interfaces 2015, 7, 17935-17946.

(31) Zhao, D.; Kim, J. F.; Ignacz, G.; Pogany, P.; Lee, Y. M.; Szekely, G. Bio-Inspired Robust Membranes Nanoengineered from Interpenetrating Polymer Networks of Polybenzimidazole/Polydopamine. ACS Nano 2019, 13, 125-133.

(32) Pérez-Manríquez, L.; Neelakanda, P.; Peinemann, K.-V. Tannin-Based Thin-Film Composite Membranes for Solvent Nanofiltration. J. Membr. Sci. 2017, 541, 137-142.

(33) Pérez-Manríquez, L.; Neelakanda, P.; Peinemann, K.-V. MorinBased Nanofiltration Membranes for Organic Solvent Separation Processes. J. Membr. Sci. 2018, 554, 1-5.

(34) Abdellah, M. H.; Pérez-Manríquez, L.; Puspasari, T.; Scholes, C. A.; Kentish, S. E.; Peinemann, K.-V. Effective Interfacially Polymerized Polyester Solvent Resistant Nanofiltration Membrane from Bioderived Materials. Adv. Sustainable Syst. 2018, 2, 1800043.

(35) Zhou, A.; Shi, C.; He, X.; Fu, Y.; Anjum, A. W.; Zhang, J.; Li, W. Polyarylester Nanofiltration Membrane Prepared from Monomers of Vanillic Alcohol and Trimesoyl Chloride. Sep. Purif. Technol. 2018, $193,58-68$.

(36) Zhang, C.; Ou, Y.; Lei, W. X.; Wan, L. S.; Ji, J.; Xu, Z. K. $\mathrm{CuSO} 4 / \mathrm{H} 2 \mathrm{O} 2$-Induced Rapid Deposition of Polydopamine Coatings with High Uniformity and Enhanced Stability. Angew. Chem. 2016, $128,3106-3109$.

(37) Wei, Q.; Zhang, F.; Li, J.; Li, B.; Zhao, C. Oxidant-Induced Dopamine Polymerization for Multifunctional Coatings. Polym. Chem. 2010, 1, 1430-1433.

(38) Luo, C.; Liu, Q. Oxidant-Induced High-Efficient MusselInspired Modification on PVDF Membrane with Superhydrophilicity and Underwater Superoleophobicity Characteristics for Oil/Water Separation. ACS Appl. Mater. Interfaces 2017, 9, 8297-8307.

(39) Jeon, Y. J.; Kang, S. M. Chemically Stable Poly (Norepinephrine) Coatings on Solid Substrates by Post-Oxidation. Polym. Degrad. Stab. 2013, 98, 1271-1273. 
(40) Wei, H.; Ren, J.; Han, B.; Xu, L.; Han, L.; Jia, L. Stability of Polydopamine and Poly (DOPA) Melanin-Like Films on the Surface of Polymer Membranes Under Strongly Acidic and Alkaline Conditions. Colloids Surf., B 2013, 110, 22-28.

(41) Zhang, C.; Lv, Y.; Qiu, W.-Z.; He, A.; Xu, Z.-K. Polydopamine Coatings with Nanopores for Versatile Molecular Separation. ACS Appl. Mater. Interfaces 2017, 9, 14437-14444.

(42) Toh, Y. S.; Loh, X.; Li, K.; Bismarck, A.; Livingston, A. In Search of a Standard Method for the Characterisation of Organic Solvent Nanofiltration Membranes. J. Membr. Sci. 2007, 291, 120125.

(43) Müller, M.; Keßler, B. Deposition from Dopamine Solutions at Ge Substrates: An In Situ ATR-FTIR Study. Langmuir 2011, 27, 12499-12505.

(44) Jeon, J.-R.; Kim, J.-H.; Chang, Y.-S. Enzymatic Polymerization of Plant-Derived Phenols for Material-Independent and Multifunctional Coating. J. Mater. Chem. B 2013, 1, 6501-6509.

(45) Ding, Y.; Weng, L.-T.; Yang, M.; Yang, Z.; Lu, X.; Huang, N.; Leng, Y. Insights into the Aggregation/Deposition and Structure of a Polydopamine Film. Langmuir 2014, 30, 12258-12269.

(46) Shao, L.; Cheng, X.; Wang, Z.; Ma, J.; Guo, Z. Tuning the Performance of Polypyrrole-Based Solvent-Resistant Composite Nanofiltration Membranes by Optimizing Polymerization Conditions and Incorporating Graphene Oxide. J. Membr. Sci. 2014, 452, 82-89.

(47) Dhand, C.; Harini, S.; Venkatesh, M.; Dwivedi, N.; Ng, A.; Liu, S.; Verma, N. K.; Ramakrishna, S.; Beuerman, R. W.; Loh, X. J.; Lakshminarayanan, R. Multifunctional Polyphenols- and Catecholamines-Based Self-Defensive Films for Health Care Applications. ACS Appl. Mater. Interfaces 2016, 8, 1220-1232.

(48) Su, L.; Yu, Y.; Zhao, Y.; Liang, F.; Zhang, X. Strong Antibacterial Polydopamine Coatings Prepared by a Shaking-Assisted Method. Sci. Rep. 2016, 6, 24420.

(49) Park, H. B.; Kamcev, J.; Robeson, L. M.; Elimelech, M.; Freeman, B. D. Maximizing the Right Stuff: The Trade-Off between Membrane Permeability and Selectivity. Science 2017, 356, No. eaab0530.

(50) Saiz, C. A.; Darvishmanesh, S.; Buekenhoudt, A.; Van der Bruggen, B. Shortcut Applications of the Hansen Solubility Parameter for Organic Solvent Nanofiltration. J. Membr. Sci. 2018, 546, 120127. 